EPJ Web of Conferences 59, 16004 (2013)

DOI: $10.1051 /$ epjconf/20135916004

(C) Owned by the authors, published by EDP Sciences, 2013

\title{
Structural properties of hydrogen isotopes in solid phase in the context of inertial confinement fusion
}

\author{
Carlo Guerrero ${ }^{1, a}$, Santiago Cuesta-Lopez ${ }^{2, \mathrm{~b}}$ and J.M. Perlado ${ }^{1}$ \\ 1 Nuclear Fusion Institute. ETSII. Polytechnic University of Madrid, Madrid, Spain \\ 2 University of Burgos, I + D + I Building, Plz. Misael Bañuelos s/n. 09001 Burgos, Spain
}

\begin{abstract}
Quality of Deuterium-Tritium capsules is a critical aspect in Inertial Confinement Fusion. In this work, we present a Quantum Molecular Dynamics methodology able to model hydrogen isotopes and their structural molecular organisation at extreme pressures and cryogenic temperatures $(<15 \mathrm{~K})$. Our study sets up the basis for a future analysis on the mechanical and structural properties of DT-ice in inertial confinement fusion (ICF) target manufacturing conditions.
\end{abstract}

\section{INTRODUCTION}

The design of the ignition and high-gain targets for inertial confinement fusion (ICF) requires a condensed uniform layer of the hydrogen fuel on the inner surface of typically a spherical polymer shell $[1,2]$. Targets proposed for the National Ignition Facility (NIF) and the HiPER campaigns, consist of small spheres of a certain material (metallic or polymer), filled with a mixture of deuterium-tritium (either solid or liquid, depending on the case), expected to be uniformly distributed, at cryogenic temperatures $(<15 \mathrm{~K})$, and named Deuterium-Tritium ice (DT-ice).

Manufactured target spheres have a diameter of 0.4 to $1.8 \mathrm{~mm}$, and are at a pressure of 25 to $1000 \mathrm{~atm}$ [3]. Recent studies show the crucial importance of the distribution of hydrogen on the surface of the fuel pellet. Such distribution is quasi-uniform, DT ice thickness varying with the solid angle [4]. Unfortunately this irregular distribution, both in thickness and nano-crystalline physical properties, is expected to play a critical influence in the implosion process with consequences in target efficiency $[3,4]$. The solid in the external layer of the DT-ice structure is known to be a molecular solid, composed of molecules as points of a crystal lattice. In principle, within the manufacturing target conditions, the hydrogen and its isotopes adopt this order for pressures below $50 \mathrm{GPa}$, and temperatures below $13 \mathrm{~K}$ [5]. Consistently with experimental studies, that depict the phase diagram of hydrogen, it is well known that, in the liquid to solid phase transition, it forms a molecular solid. Depending on pressure, it can be organised according to a hexagonal close-packing (HCP) configuration (Phase I) [5, 6]. However, as pressure increases over $100 \mathrm{GPa}$ (remaining at low temperature), hydrogen goes onto two different phase transitions (Phase II, III). In phase II (or the broken symmetry phase) the structure is possibly $\mathrm{Pa} 3$, and the rotation is hindered but is nevertheless of quite wide angle. In phase III (also called the HA phase) the structure is predicted to be in the orthorhombic class and there is still wide-angle libration [7]. In this work, we would like to raise the attention of the ICF community on this matter. This complex structural scenario should be taken into account at the level of implosion efficiency considerations.

\footnotetext{
ae-mail: guerrerocarlo@yahoo.es

be-mail: scuesta@ubu.es
}

This is an Open Access article distributed under the terms of the Creative Commons Attribution License 2.0, which permits unrestricted use, distribution, and reproduction in any medium, provided the original work is properly cited. 

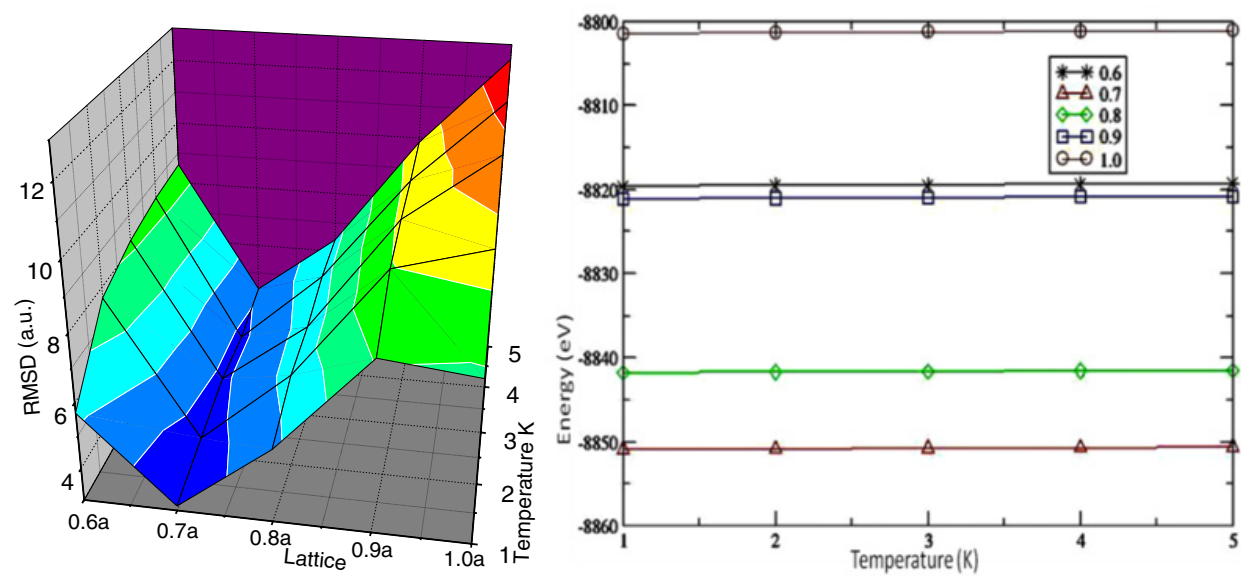

Figure 1. Selection criteria of the lattice parameter for our initial HCP hydrogen crystal. Different lattice constants $(0.6 a, 0.7 a, 0.8 a, 0.9 a \mathrm{y} 1.0 a)$ where evaluated at five temperatures ranging from $1 \mathrm{~K}$ to $5 \mathrm{~K}$. (left) RMSD difference between the initial and final structural configuration after $2000 \mathrm{fs}$ of QMD structural relaxation. (right) Energy comparison of each structural state and lattice parameter after relaxation.

On the other hand, although different authors have proposed different phase diagrams for pure hydrogen (H) and deuterium (D) [2, 8-10], only a few studies can be found in the literature for pure tritium (T). Moreover, there is a considerable lack of information about mixtures of the three isotopes HD, TD, HT, particularly at cryogenic temperatures (below $10 \mathrm{~K}$ ). Therefore, it is of general scientific interest, and particular to the Fusion community, to try to fill this gap as soon as possible.

\section{METHODOLOGY TO SIMULATE THE MOLECULAR STRUCTURE OF H ISOTOPES IN AN ICF CONTEXT}

Our methodology is based on a Quantum Molecular Dynamics (QMD) approximation of the evolution of a small orthorhombic cluster of hydrogen atoms, sampled in the NPT statistical physics ensemble (constant Pressure, Temperature and Number of atoms). The system was coupled to both a barostat and a thermostat, in order to take into account thermal fluctuations, and to equilibrate states at every fixed pressure. Note that initially, it is critical to find a stable structural starting state. To that end, the solid molecular hydrogen was introduced and arranged according to a HCP structure. We defined the morphology of the solid, the orientation of the molecules, and eventually the distribution of different isotopes that may compose the crystal structure. The system considered was a HCP structure with 576 atoms (288 molecules of hydrogen) and an orientation of all molecular axes in the $\mathrm{z}$ direction. In order to optimize the lattice parameter, we have explored both structural and energy phase spaces through QMD simulations at five different constant temperatures (from $1 \mathrm{~K}$ to $5 \mathrm{~K}$ ) and tested several lattice parameters $(1.0 a, 0.9 a, 0.8 a, 0.7 a, 0.6 a)$. The initial lattice parameter selected was: $\mathrm{a}=3783 \AA$ [11], reported experimentally by Galtsov et al in similar working conditions. QMD simulations were carried out by means of SIESTA code [12]. The quest for a stable system structure was defined allowing the system to evolve until a temperature variation of less than $1 \mathrm{~K}$ is reached. Pressure fluctuations were tolerated in a range of $\pm 0.5 \mathrm{kbar}$.

In this first step, selection of the most stable state, and thus the most optimal lattice parameter, was done paying attention to the energy levels of each of the structures (Figure 1, right). Complementarily, global distortion was monitored by calculation of the root-mean-square deviation (RMSD) in displacement of the atoms with respect to their initial positions (Figure 1, left). Note that the thermal variation that we have explored, allows a better exploration around the minima. Finally, the slightest 


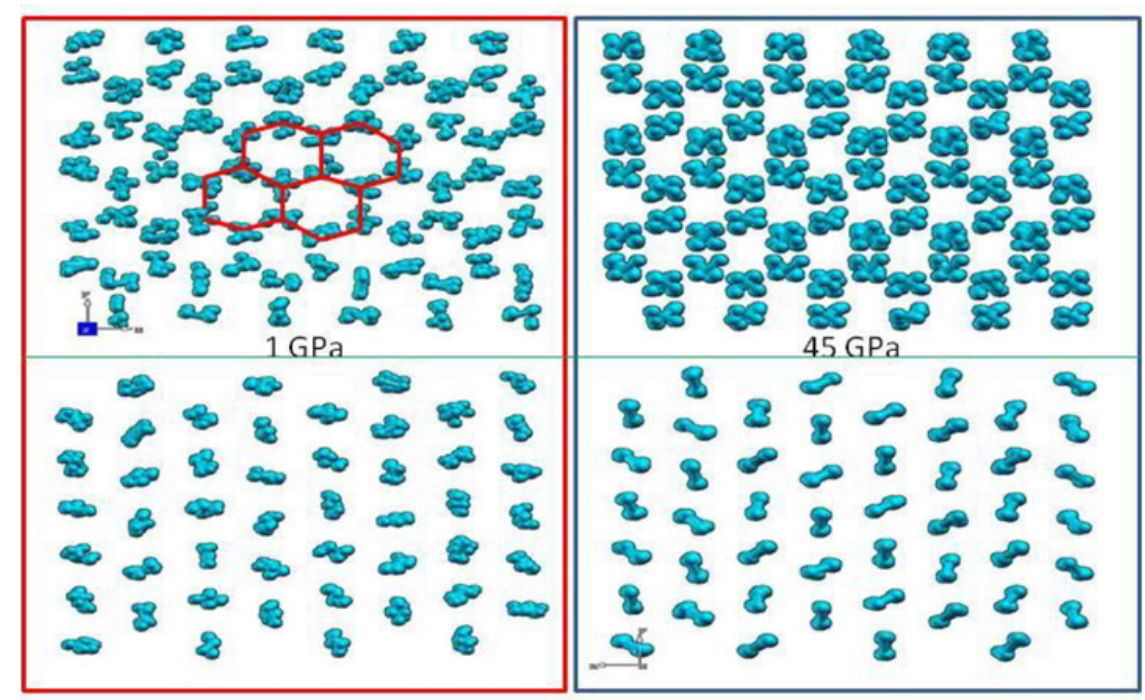

Figure 2. Orientation of the molecules for the solid hydrogen structure in our computational test, at temperature $15 \mathrm{~K}$, and $1 \mathrm{ps}$ of a QMD trajectory. Two characteristic pressures are shown: $1 \mathrm{GPa}$ (left) and $45 \mathrm{GPa}$ (right). The HCP hexagonal structure of Phase I is highlighted in red (left). Structurally speaking, the phase transition can be well distinguished paying attention to the differences in the arrangement of the molecules at $1 \mathrm{GPa}$ (single molecules as rotors), and the arrangement at $45 \mathrm{GPa}$ (the distribution is aligned and the rotation are restricted), in agreement with the results of Toledano et al [13].

displacement, independently of temperature, was obtained for a lattice constant of $0.7 a$, which appoints at the same time to the minimal energy state (Figure 1, right). According to these facts, an HCP $(0.7 a)$ has been selected as the initial structural starting point of every QMD trajectory that we will generate at constant pressure and temperature. QMD simulations where done using the SIESTA code (based on DFT). The following parameters were used: Hydrogen pseudopotential generated of the Troullier-Martins type, the electronic wavefunctions expanded in plane have been with a wave energy cut-off of 100 Ry. Temperature coupling was achieved using the Nose-Hoover thermostat. The coupling constant to heat bath was $100 \mathrm{Ry} \times \mathrm{fs}^{2}$. For pressure control, we decided to apply the Parrinello-Rahman barostat, with a coupling of $100 \mathrm{Ry} \times \mathrm{fs}^{2}$. Integration time step was set as $1 \mathrm{fs}$. The number of atoms for all simulations amounts to 576 atoms inside a unit box of dimensions: $19.36 \AA \times 17.88 \AA \times 20.71 \AA$.

\section{RESULTS AND VALIDATION OF METHODOLOGY}

We have carried out a detailed structural study of the solid-phase hydrogen evolution at different pressures, from $1 \mathrm{GPa}$ to $80 \mathrm{GPa}$. Simulations were performed at a fixed temperature near the triple point $(15 \mathrm{~K})$, and at desired pressures allowing a variation of not more than $\pm 1 \mathrm{GPa}$. In this part of the process, pressure was controlled by a Parrinello-Rahman barostat. Note that according to the NPT formalism, the solid volume varies; therefore periodic boundary conditions were applied.

A clear validation test of our procedure is the study of the structural changes occurring to our solid hydrogen structure as soon as pressure is raised to a point where a phase transition may occur. Comparison is possible through the theoretical model and predictions from Toledano et al [13]. Different experimental works discuss the transition from Phase I to Phase II of the hydrogen, that jumps from a configuration with molecules as free rotors (the atoms can describe a sphere), to an order where such rotations are restricted $[7,15,16]$. Toledano and collaborators predict that such a transition occurs around $110 \mathrm{GPa}$. However, taking into account the two forms of hydrogen, para (the spins of the two 


\section{EPJ Web of Conferences}

protons are antiparallel) and ortho (the spins of the two protons are parallel), a notable difference in the phase diagram can be observed [16]. Note that, below $25 \mathrm{~K}$, concentration of para-hydrogen is greater than 95\% [17], which is the ICF-target conditions case. In those conditions, the phase diagram for hydrogen shows a Phase I - Phase II transition around $40 \mathrm{GPa}$ [16], which is just the transition that we obtain after following our QMD trajectories. The pressure induced phase transition observed with our QMD assay is described in Figure 2. It shows four images: bottom pictures are projections for all the molecules on the YZ plane, where you can observe a much more pronounced phase difference for the $1 \mathrm{GPa}$ test. In that case, (Figure 2, left), projection is a circle, ie the molecules are free rotors, while in the case of $45 \mathrm{GPa}$ (Figure 2, right), there is an alignment of molecules with restricted rotation, according to the image proposed by different authors $[7,13]$. Top of the figure shows the projections on the XY plane. Again phase differences and changes in molecular order can be observed.

The reproduction of the pressure induced Phase I - Phase II transition for a molecular structure made of para-hydrogen at cryogenic temperatures, and very high pressures, here reported, represent a clear validation of our methodology, and opens the possibility of application of this protocol to get insight about the structural properties of DT in extreme conditions.

\section{CONCLUSIONS AND FUTURE WORK}

The study of hydrogen and its isotopes (deuterium and tritium, and combinations thereof), at high pressures and very low temperatures, is critical for the development of Fusion technology, in particular in the ICF target technology area. In addition, the possibility to study small hydrogen clusters and their size effects, represents a wealth of information about the way in which hydrogen interacts or can be captured by other materials. This is also crucial in other subjects concerning Fusion technology, like Tritium breeding processes or materials resistance (radiation damage) studies. Moreover, the present structural study of hydrogen isotopes near the triple point, is a big step to explore the phase diagrams for combinations of Deuterium-Tritium, absent in the literature, and of great importance not only for Fusion Science, but also in other areas like astrophysics, superconductivity, crystallography, energy storage, or rocket fuel [10].

Of further importance, in the short term, we expect by means of this methodology to get insight of the physical and mechanical properties that this quasi-crystalline structure may impose over the shock propagation in the implosion process.

S. Cuesta-López would like to thank Prof. Elena Koresheva (Russian Academiy of Sciences) and Prof. Martin Tolley (STFC Central Laser Facility, UK) for fruitful scientific discussions about ICF targets. The authors are in debt with the HiPER project (HiPER - Fp7-infraestructures-2007-1. capacities workprogramme Grant Agreement No. 211737) which is funding C. Guerrero. We also acknowledge the creators of SIESTA code, the main tool used in the studies here presented.

\section{References}

[1] I V Aleksandrova et al., Journal of Physics D: Applied Physics, 37, 1163-1178 (2004)

[2] H. Kawamura et al., Review of High Pressure Science and Technology, 18, No. 1 (2008)

[3] I.V Alekxandrova et al., Journal of Russian Laser Research, Volume 28, Number 3 (2007)

[4] D. R. Harding et al., LLE Review, Quarterly Rept., 99, 160 (2004)

[5] P.C. Souers, Hydrogen Properties for Fusion Energy. University of California.(1986)

[6] A. Chijioke and F. Silvera, Phys. Rev. Lett. 97, 255701 (2006)

[7] B. Edwards and N.W. Ashcroft, PNAS, 101, 12, 4013 (2004)

[8] I.F. Silvera et al. Journal of Physics: Conference Series, 215, 012194 (2010) 


\section{IFSA 2011}

[9] M. I. Eremets and I. A. Trojan. JETP Letters, 89, No. 4, pp. 174-179 (2009)

[10] A. Goncharov, R. J. Hemley, and E. Gregoryanz, Phys. Rev. Lett. 102, 149601 (2009)

[11] N.N. Galtsov et al., Fizika Nizkikh Temperatur. 29, Nos.9/10, p. 1036-1040 (2003)

[12] SIESTA code: J.M. Soler et al. J. Phys.: Condens. Matter 14, 2745-2779 (2002)

[13] P. Toledano et al., Phys. Rev. Lett. 103, 105301(2009)

[14] G.W. Collins, W.G. Unites, E.R. Mapoles and T.P. Bernat, Phys. Rev. B, 53, 1, 102 (1996)

[15] A. Goncharov et al., Phys. Rev. B, 54, 22, R15590 (1996)

[16] Mazin et al., Phys. Rev. Lett. 78, Number 6 (1997)

[17] I. Silvera, Rev. Mod. Phys. 52, 2, Part I (1980) 